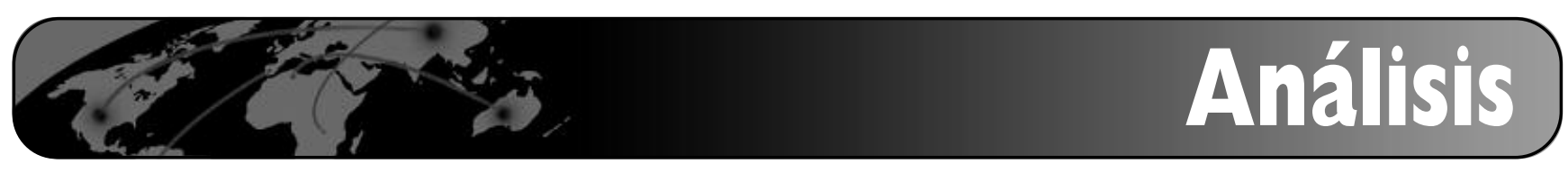

\title{
Descripción de un sistema de información gubernamental: Argos
}

\author{
Por Lourdes Castelló Cogollos
}

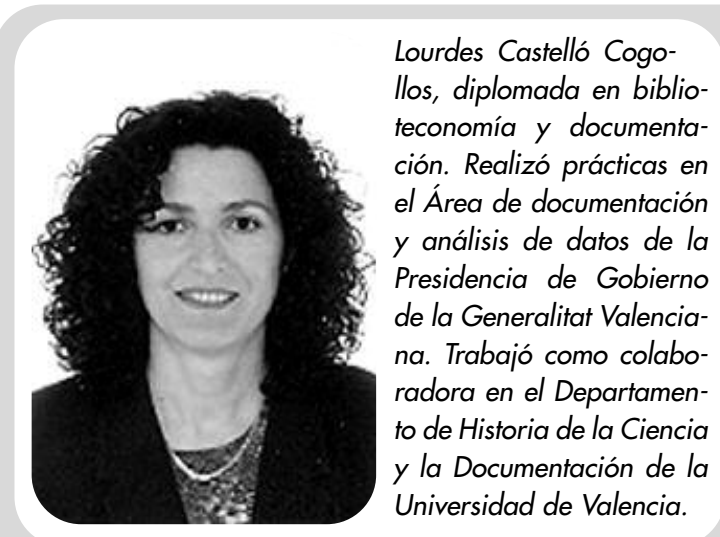

Resumen: Se expone el funcionamiento de las unidades de información documental (UIDs) en entornos gubernamentales, en las cuales se trabaja con herramientas complejas de gestión, los sistemas de información documental (SID). La autora describe el proyecto Argos, desarrollado en el área documental de la Presidencia del Gobierno de la Comunitat Valenciana. A partir de este caso se remarca la función del documentalista en instituciones públicas. En estos ámbitos, las UIDs se organizan en subdepartamentos especializados que se responsabilizan de metas concretas en la misión general. Por otra parte, se consideran los SIDs como espacios virtuales de trabajo multidisciplinar en los que convergen tanto los profesionales como los usuarios.

Palabras clave: Unidad de información y documentación, Sistema de información documental, Instituciones gubernamentales, Entornos virtuales de gestión documental, Argos, Comunitat Valenciana.

\section{Title: Description of a governmental information system: Argos}

Abstract: This article describes how information and documentation units (UIDs) work in a governmental environment. These professional teams use complex management tools known as information and documentation systems (SIDs). The author describes the case of the Argos project, a system developed by the documentation area of the Presidential Department of the Valencian Community government. This study leads to a discussion of the functions of document specialists in public institutions. In these environments, UIDs are organised into specialised sub-departments responsible for specific goals that meet the overall mission of the organisation. For their part, SIDs are virtual and multidisciplinary workspaces where professional staff and users come together.

Keywords: Information and documentation department, Information and documentation system, Governmental institutions, Virtual environment of document management, Argos, Valencian community.

Castelló Cogollos, Lourdes. «Descripción de un sistema de información gubernamental: Argos". En: El profesional de la información, 2006, mayo-junio, v. 15, n. 3, pp. 202-208.

\section{Introducción}

VIVIMOS EN UNA SOCIEDAD en la que la información ha alcanzado un valor significativo. Lo que se pretende es crear conocimiento por medio de la máxima explotación de los datos. El conocimiento se convierte así en factor indispensable a la hora de decidir, actuar y contribuir al progreso social.

El rápido crecimiento de la información es también uno de nuestros principales inconvenientes ya que su exceso genera ruido mientras que una acotación excesiva puede producir silencio (hará perder información importante). Esto hace imprescindible una gestión adecuada de los datos con el objetivo de ofrecer la mayor calidad posible a los responsables de la toma de decisiones en las organizaciones.

En los gobiernos la toma de decisiones es muchas veces a nivel estratégico y a largo plazo, con lo que el valor de la información es aún mayor. Este tipo de organizaciones maneja gran cantidad de datos que se deben analizar, sintetizar y explotar adecuadamente con el objetivo de optimizar la gestión pública.

En este artículo se resalta la importancia de las unidades de documentación como gestoras de la información y por tanto del conocimiento en organismos gubernamentales. Para ello, en la primera parte del artículo se muestra en qué consisten teóricamente estas unidades y en la segunda se describe el proyecto Argos, circunscrito al Área de documentación y análisis de datos 


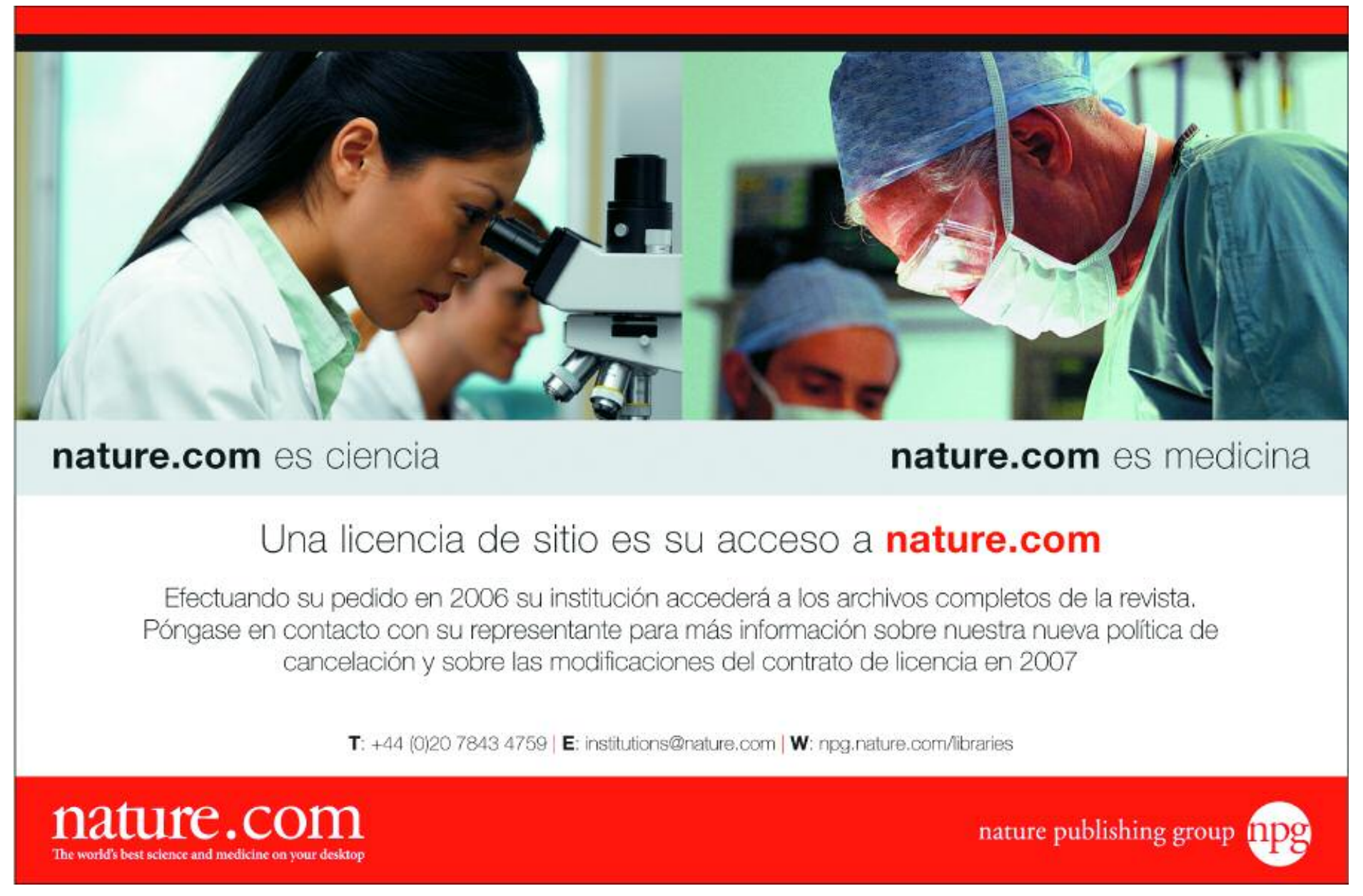

de la Presidencia de la Generalitat Valenciana.

\section{Unidades de documentación y sistemas de información}

La unidad de información y documentación (UID) es el departamento que se encarga de gestionar la información, y por tanto el conocimiento, dentro de la organización. Para su trabajo es imprescindible la investigación y la innovación de los procedimientos y los instrumentos necesarios a fin de ofrecer inteligencia competitiva al usuario (Navarro; Esteban, 2004).

La inteligencia competitiva tiene como tareas imprescindibles estudiar el entorno para identificar, seleccionar, almacenar, analizar, interpretar y difundir información de importancia estratégica (Hiebaum). Esta información se clasifica en ambiental o externa (procedente del entorno con el fin de de- terminar qué necesita el "mercado"); interna (producida por la propia empresa y producto del proceso de la información externa que servirá para la toma de decisiones) y corporativa (que la empresa o organización proyecta al exterior) (Chain, 2000).

«La gestión de la
información es cada
vez más valorada por
los dirigentes y
responsables de las
instituciones
gubernamentales ya
que les permite tomar
decisiones bien
informadas»

La UID debe recopilar y organizar esa información de modo que se pueda acceder a ella fácilmente y garantizar que cualquier usuario, tanto real como potencial, pueda disponer de los datos necesarios cuando realmente los requiera: lo que se conoce como "valor de oportunidad". Finalmente, estas unidades deben promocionar el uso correcto de los recursos informativos disponibles y con ello intentar que los usuarios puedan ser cada vez más independientes a la hora de buscar información (Lorenzo; Miralles).

Para poder realizar estas tareas las UIDs utilizan sistemas de información documental (SID), los cuales se componen de personas, recursos materiales y procedimientos técnicos encaminados a transformar los datos en información disponible para el usuario. Son sistemas complejos con capacidad para adaptarse al entorno que les rodea, por lo que para su implantación se requerirá analizar tanto los datos como el contexto, es decir, realizar un estudio de las necesidades y usos de in- 
formación de los "clientes" a los va dirigido este sistema.

Un SID racionaliza todos los flujos de información de una organización y se compone de tres fases básicas: una entrada o input, que son los datos y documentos que se adaptan al perfil de usuario de la organización, el tratamiento de dicha documentación y una salida u out$p u t$, que sería la información ya clasificada y disponible para que sea utilizada, ya que la información sólo tiene valor si es usada.

En el tratamiento se realizan diversas tareas: catalogación, ordenación, análisis, sintetización... En definitiva todos los trabajos propios de la documentación para que la información pueda ser recuperada por parte del usuario, de forma pertinente.

Cuando se plantean los SIDs se deben tener en cuenta las características de los usuarios a los que va dirigido, de cuya participación e integración dependerá su éxito. En este contexto se pueden distinguir los siguientes tipos y niveles de usuarios:

-Directos: por un lado existen los gestores del sistema, que lo usan y a su vez lo alimentan; y por otro la población diana a la que va dirigido y que sabe utilizarlo para su trabajo.

-Secundarios: son los intermediarios de la información, es decir, los que interactúan con el sistema para preparar informes pero no utilizan los resultados directamente para su trabajo.

-Indirectos: beneficiarios o consumidores de la información que utilizan el output generado por un equipo de trabajo y confían en el proceso de filtro y en la capacidad de análisis de las personas que lo integran (Felip, 1999).

Las organizaciones gubernamentales (gabinetes de presidencia, diputaciones, ministerios, etc.), re-

\section{Esquema de flujos de información gubernamentales}

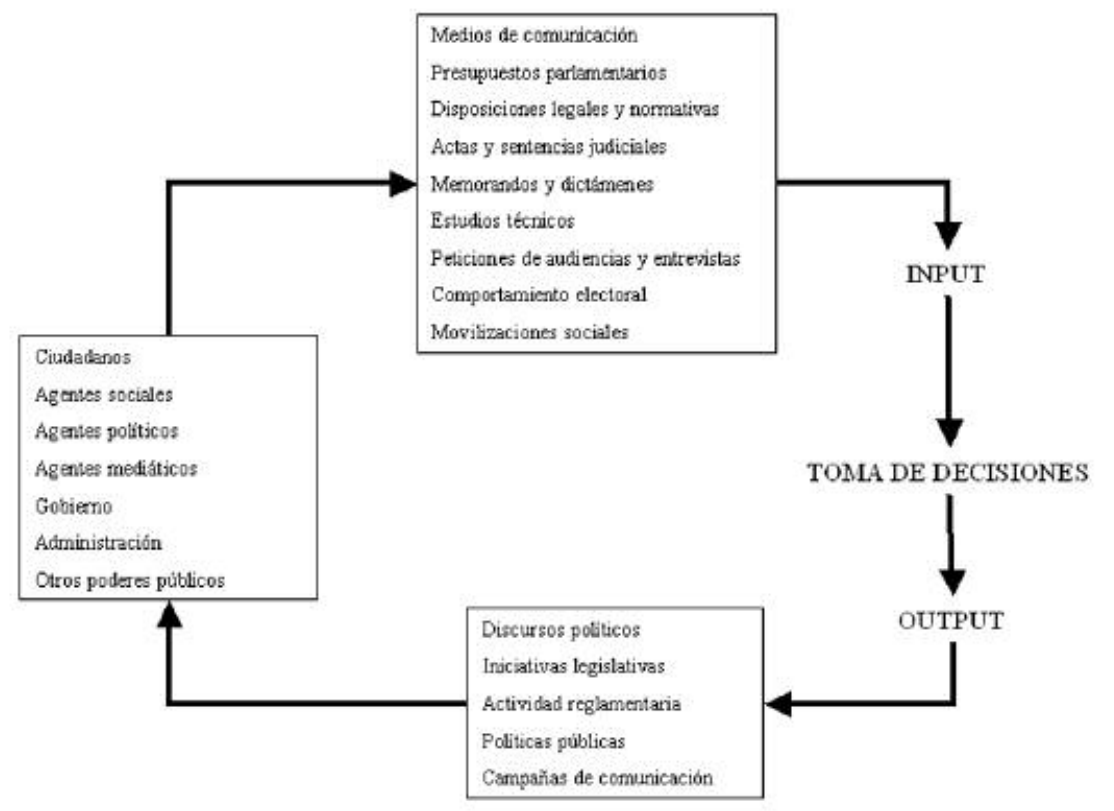

Figura 1

quieren que la información sea gestionada eficazmente. La que consumen es muy diversa y procede de distintos ámbitos: de la propia administración, de los ciudadanos, de los agentes mediáticos, políticos, culturales y económicos, etc. Con estos tipos y procedencias, el ciclo de información quedaría establecido de la forma que se muestra en la figura 1.

Desde la segunda legislatura (1987-1991) la Generalitat Valenciana ha puesto en funcionamiento un departamento específico que desarrolla un sistema de información con el objetivo de racionalizar y optimizar los flujos de comunicación. Éste es un caso ilustrativo de la necesidad de una UID en un contexto de alta responsabilidad, por lo que hemos considerado que su análisis y descripción es de interés para la comunidad profesional de documentalistas. También puede ser útil para que los responsables políticos sean conscientes de la importancia de disponer de un equipo profesional y de una organización eficaz en el ámbito de la información.

\section{Análisis de caso: el proyecto Argos}

Desde su creación, el Área de documentación y análisis de datos de la Generalitat Valenciana ha dependido de diversas subsecretarías, hasta que en el año 2004 pasó a formar parte del Gabinete de Presidencia. En ella trabajan estadísticos, economistas, documentalistas, sociólogos, juristas, politólogos, además del personal administrativo y de servicios. O sea, los equipos de trabajo en una UID son multidisciplinares, ya que las tareas a realizar requieren especialización. Como veremos el SID es a la vez una herramienta de trabajo y un punto de encuentro de todos los profesionales involucrados en la consecución de objetivos.

La especificidad de las tareas ha llevado a la dirección a estructurar el equipo en diversas unidades: Análisis de datos, Documentación general, Estudios sociológicos, Análisis de programas y Análisis de políticas públicas. Como vemos, pues, la especialización conlleva que las UIDs complejas queden organizadas en subdepartamentos que 
se responsabilizan de la consecución de las distintas metas y objetivos determinados por ley:

-Misiones: asesoramiento al Presidente de la Comunidad Valenciana y apoyo a la toma de decisiones de la Presidencia.

-Metas: realización de análisis y previsión a largo plazo, así como soporte técnico documental.

-Objetivos: producción de informes, previsiones económicas y financieras, análisis de comunicación y previsión de escenarios demoscópicos. También debe realizar y mantener diversas bases de datos (territoriales, estadísticas, documentación del Consell, archivo histórico, etc.).

Aunque estos objetivos están enfocados a la figura del Presidente, el área ofrece otros muchos servicios de apoyo a usuarios internos y externos. Esto nos indica que, en general, las UIDs pueden tener delimitados unos objetivos concretos pero acaban desarrollando y ofreciendo otros servicios que van más allá, siempre dirigidos a mejorar la gestión y el trabajo de todos los miembros del gobierno y hasta de usuarios externos (investigadores, medios de comunicación o ciudadanos). Este grado de acceso es inherente a su condición de servicio público.

\section{«La especialización conlleva que las UIDs complejas queden organizadas en subdepartamentos que se responsabilizan de la consecución de distintas metas y objetivos de la misión que atañe a todo el departamento»}

El Área de documentación y análisis de datos creó el proyecto Argos para el cumplimiento de estas misiones, metas y objetivos. Se trata de un SID que reúne todas las bases de datos, consultables en una

\begin{tabular}{|c|c|c|}
\hline Secciones principales & Público & Privado \\
\hline \multirow[t]{2}{*}{ Secciones } & \multicolumn{2}{|l|}{$\begin{array}{l}\text {-Información electoral } \\
\text {-Información demoscópica } \\
\text {-Información periodística } \\
\text {-Información parlamentaria } \\
\text { - Información municipal } \\
\text {-Información bibliográfica } \\
\text {-Información legislativa } \\
\end{array}$} \\
\hline & & -Quién es quien \\
\hline El presidente & & $\begin{array}{l}\text { - Sibil la web } \\
\text {-Viajes al extranjero } \\
\text {-Entrevistas y artículos de } \\
\text { opinión } \\
\text {-Comparecencias } \\
\text { parlamentarias }\end{array}$ \\
\hline Petición de información & & $\begin{array}{l}\text {-Desiderata } \\
\text { - Reprografía } \\
\text {-Solicitud de información }\end{array}$ \\
\hline \multirow[t]{2}{*}{ Recursos } & \multicolumn{2}{|c|}{$\begin{array}{l}\text { - Archivo electoral } \\
\text { - Gavina web } \\
\text { - Busca'm } \\
\text { - Bolsum } \\
\text { - Demoscòpia } \\
\text { - Revista valenciana d'estudis autonòmic } \\
\end{array}$} \\
\hline & & $\begin{array}{l}\text { - Casandra } \\
\text { - Banco de datos municipal } \\
\text {-Excalibur }\end{array}$ \\
\hline
\end{tabular}

Tabla 1. Esquema de Argos única interfaz, y otros recursos y servicios. Desde su nacimiento ha ido cambiando de sistema de gestión e incorporando nuevas fuentes informativas, todo lo cual ha propiciado también una renovación de su imagen.

La tendencia actual en las instituciones gubernamentales en general es la incorporación de software de código abierto. Así pues, las UIDs también aprovechan estos productos y ayudan a promover el movimiento open source desarrollando incluso nuevos módulos, por lo que otras instituciones públicas (bibliotecas, universidades, organismos) pueden beneficiarse de este trabajo en común.

Durante 2005 se implantó Typo3-CMS (content management system), basado en código libre (open source), con el cual se gestiona la web permitiendo tener más autonomía en la creación de documentos, hacer cambios en los contenidos en tiempo real, reducir los tiempos de publicación, gestionar contenidos multi-idioma, y mejorar la navegación y la capacidad de crecimiento del sitio web (figura 2).

Con Typo3-CMS se creó una nueva interfaz de la sede web en la que en un menú lateral de la izquierda se pueden consultar todos los servicios y bases de datos, divididos en catorce secciones. En el menú de la derecha se encuentran estudios de opinión, y otros temas y documentos de interés general de la GVA. En la parte superior se enlaza al mapas de la web, información sobre el Área, FAQ, etc.

A efectos de acceso, Argos se divide en dos niveles: una parte externa, para usuarios públicos, y otra interna o privada, para los de la Administración (tabla 1), dependiendo del nivel de sensibilidad y de confidencialidad de la información. En toda UID se tiene que aplicar una política de segmentación a estos dos niveles. 


\section{Secciones y servicios más importantes}

En la intranet:

\section{—Quién es quien}

Ofrece información bio-bibliográfica sobre los miembros del gobierno autonómico y central de la legislatura actual así como de las anteriores.

\section{-Presidente}

Recopila toda la información del/para el Presidente de la Generalitat Valenciana, y da acceso a la base de datos Sibil.la, la cual se ha convertido en el sistema más importante de gestión de información política de actualidad sobre la Comunidad Valenciana. Contiene más de 175.000 registros en los que se analizan documentalmente más de 250.000 páginas de los diarios valencianos y de los principales periódicos de información general de difusión española. Con Sibil.la web, todos los interesados pueden obtener tanto el producto del análisis realizado por los documentalistas sobre la información de prensa como los facsímiles digitalizados de los originales (sección Monogràfics Sibil.la web).

-Archivo histórico de resultados electorales

Resultados de elecciones españolas, de todas las comunidades autónomas celebradas desde 1977, y del Parlamento Europeo. Para la Comunidad Valenciana se tiene acceso además a los datos comarcales y municipales.

\section{- Gavina web}

Información no periodística: monografías, revistas especializadas, referencia, fuentes legales y estadísticas, informes de circulación restringida, bases de datos y webs. Es el catálogo de la biblioteca departamental del Gobierno valenciano.

\section{-Busca'm}

Sistematiza el gran volumen de fuentes en la Web relacionados con cualquiera de los ámbitos de interés de las administraciones y políticas públicas, identificadas y seleccio-

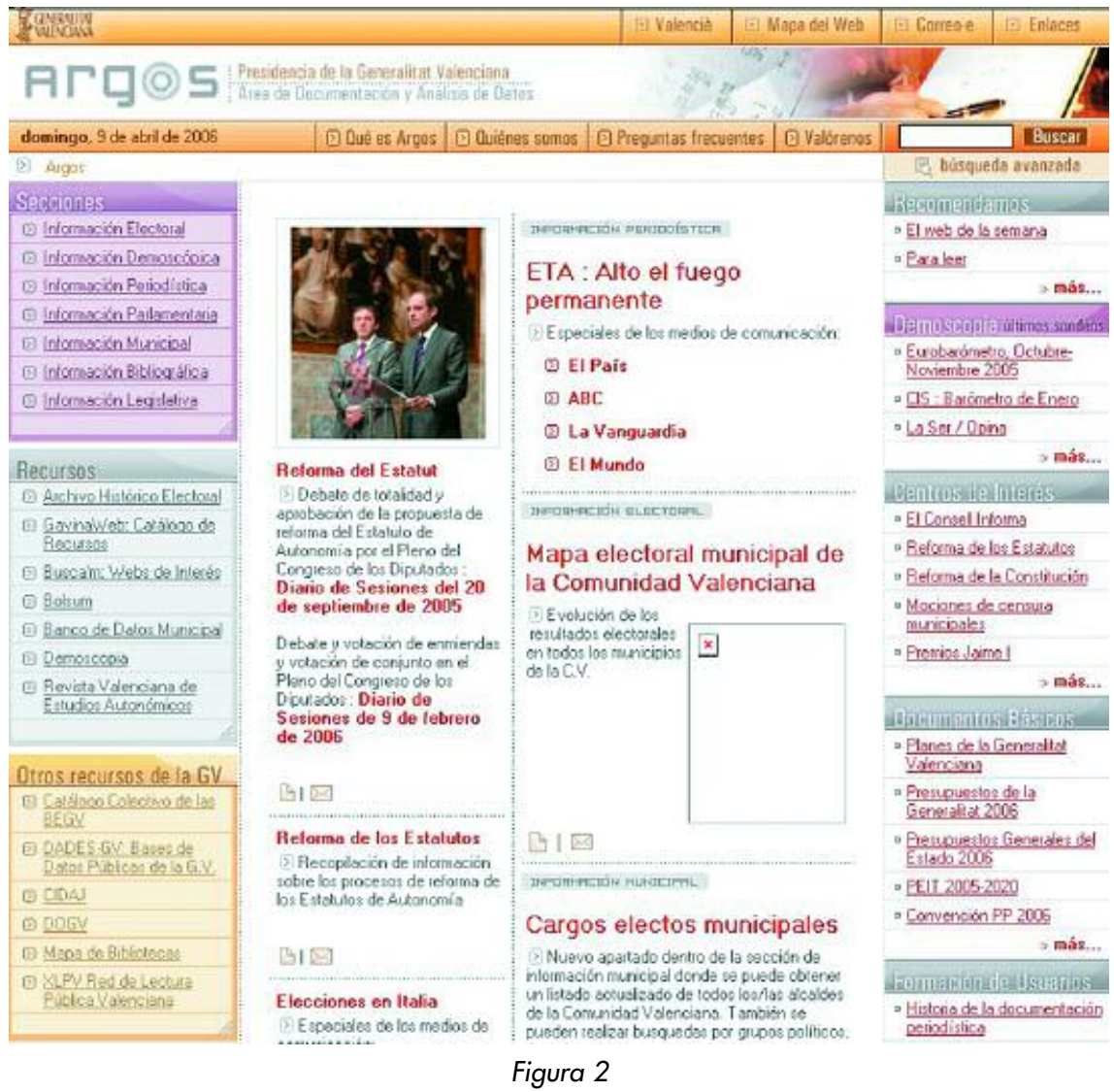

nadas por el Centro de Información y Documentación Jurídico-Administrativa (Cidaj) y por la Unidad de Documentación General de Presidencia, ambos de la Generalitat Valenciana.

\section{- Casandra}

Sondeos de opinión pública realizados por la Presidencia de la Generalitat Valenciana desde 1984.

\section{-Banco de datos municipal}

Datos sobre municipios y comarcas de la Comunidad Valenciana agrupados por equipamientos, infraestructuras, actuaciones del Consell, etc., desde 1995.

http://www.gva.es/cidaj/val/index0.htm

http://www.pre.gva.es/argos/do cus/public_val/index.html

\section{-Excalibur}

Información periodística seleccionada por la Unidad de Documentación General de Presidencia desde abril de 2000. Recoge miles de documentos aparecidos en la prensa sobre temas de interés de ámbito autonómico, español e internacional. Es un servicio complementario a la base de datos Sibil.la. El resultado de las operaciones de los usuarios son dos tipos de archivo para cada noticia: por un lado, uno de texto con el contenido del documento; por otro, un archivo de imagen, producto de la digitalización del original.

\section{-Bolsum}

Servicio de alerta de las publicaciones periódicas especializadas recibidas. El equipo de trabajo puede encontrar sumarios de revistas, solicitar copias de artículos, referencias bibliográficas y otros documentos de apoyo.

\section{—Demoscòpia}

Estimaciones de voto a partir de la información publicada en la prensa nacional y valenciana, desde 


\section{Facilitando el acceso integrado a contenidos}

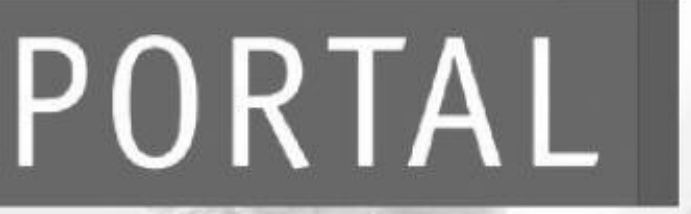

ZPORTAL facilita un entorno de búsqueda federada que lanza búsquedas a diversos recursos de información de forma simultánea, recuperando los resultados en una única pantalla.

Con ZPORTAL se ha desarrollado el portal de salud del Reino Unido, www.library.nhs.uk, la herramienta que personal sanitario, documentalistas, personal de administración y pacientes utilizan para la búsqueda integrada de información.

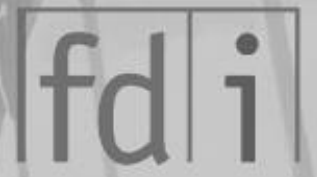

fretwell-downing informatics

telephone: +441142816040 email: enquiries@fdisolutions.com www.fdisolutions.com
Distribuidor en España:

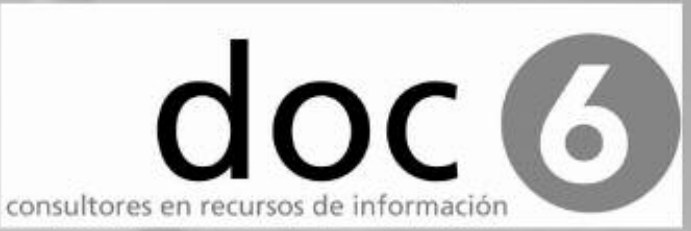

Mallorca, 272, planta 3 - 08037 Barcelona Tel. 932154313 Fax: 934883621

Orense, $14,5^{\circ} \mathrm{A}-28020$ Madrid Tel. 915535207 Fax: 915346112 mail@doc6.es -www.doc6.es 
las elecciones autonómicas de 1995 hasta la actualidad en el primer caso, y desde las elecciones generales de 1996 hasta la actualidad en el segundo.

\section{- Revista valenciana d'estudis} autonòmics.

Como vemos, la información que recogen las UIDs gubernamentales es de carácter político-electoral, económico y legislativo, aunque esto depende del tipo de organización a la que pertenezca. En cada caso ponen en marcha un SID que se ajuste a la especialización de la institución a la que están adscritas. El elemento transversal a todas ellas es que existe una información base (legislación, normativas, manuales de catalogación y de bases de datos, documentos internos de trabajo) y otra más especializada en la temática concreta (sanidad, educación, medio ambiente, política, economía, etc.).

\section{Conclusiones}

Se ha resaltado la importancia que han alcanzado las unidades de información y documentación (UIDs) en el funcionamiento de las organizaciones. Vemos como en ellas los profesionales se afanan en contribuir al éxito de la institución para las que trabajan.

Nuestra impresión es que la gestión de la información es cada vez más valorada por los dirigentes y responsables de las instituciones gubernamentales, ya que la necesitan si quieren tomar decisiones óptimas. Se han expuesto las características fundamentales de las UIDs dependientes de instituciones públicas, y se ha explicado cómo utilizan unas herramientas complejas de gestión que son los sistemas de información documental (SID).

Hemos analizado el funcionamiento de un SID con la descripción de Argos, desarrollado por el Área de Documentación y Análisis de Datos de la Presidencia de la

Generalitat Valenciana. El estudio del caso nos aporta nuevas ideas sobre cómo operan las UIDs, que en estas organizaciones, dada su complejidad, se ramifican en diversos subdepartamentos donde trabajan profesionales con perfil especializado en varias disciplinas. De esta forma, además de ser una herramienta, se convierte en un punto de encuentro donde convergen tanto los técnicos como los usuarios (internos y externos). Pasa a ser un "entorno virtual de comunicación y trabajo".

\section{«El sistema de información y documentación (SID) pasa a ser, además, un 'entorno virtual de comunicación y trabajo'»}

Como en la distribución física de una institución pública, el SID también requiere unos espacios públicos, en los que se atiende y se informa a los usuarios externos, y otros privados, para uso de los trabajadores en la administración. El carácter de privacidad de estas secciones viene dado por el grado de sensibilidad y confidencialidad de la información que se trata en ellos.

Para implantar estos sistemas se han de realizar no sólo estudios de las necesidades de los usuarios (reales y potenciales), sino también del entorno que les rodea. Su éxito también depende de su adaptabilidad a las nuevas exigencias y necesidades cambiantes. En las mejoras que se implantan el acento se pone en la usabilidad y navegabilidad del sistema con el fin de que los usuarios puedan ser autónomos. En este contexto, los documentalistas, ocupan un lugar estratégico, ya que de su tarea de proporcionar inteligencia competitiva dependerá el éxito de la organización.

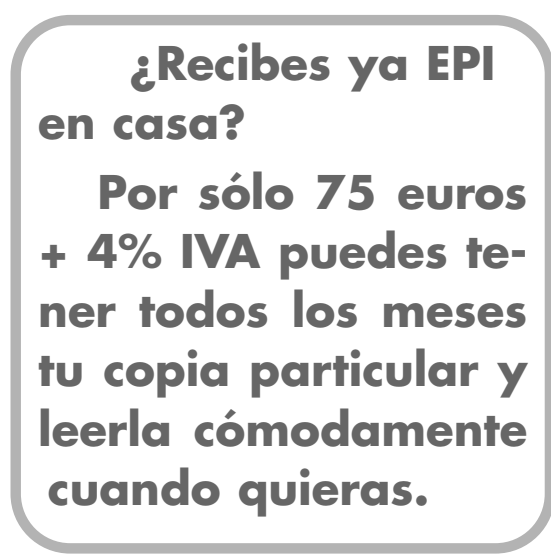

\section{Bibliografía}

Área de documentación y análisis de datos. Gabinet de Presidència de la Generalitat Valenciana. Proyecto Argos. Consultado en: 05-07-05. http://www.pre.gva.es/argos

Chaín Navarro, Celia. Gestión de la información en las organizaciones. ICE. Murcia: Universidad de Murcia, 2000.

Abad García, María Francisca; Felip i Sardà, Josep Maria; Lorenzo Gorriz, Antonio, M. "Evaluación del sistema de información Argos para la toma de decisiones en la Presidència de la Generalitat Valenciana". En: Revista valenciana d'estudis autonòmics, 1999, v. 28, pp. 205-244.

Debons, A.; Horne, E.; Cronenweth, S. Information science: an integrated view. Boston, MA: Hall \& Co., p. 14, 1988.

Generalitat Valenciana. Conselleria d'Economia, Hisenda i Ocupació. Projecte de Pressupostos. Valencia: Generalitat Valenciana, 2005.

Hiebaum, Karin. Una visión general de la inteligencia competitiva. Consultado en: 05-07-05.

http://www.gestiopolis.com/recursos $2 /$ document os/fulldocs/ger/intelcompecar

Lorenzo, Antonio; Miralles, Rosa. Servicios de documentación en entornos gubernamentales: estudio de un caso. Consultado en: 02-07-05.

http://www.hipertex.net/web/pag234htm

Moscoso, Purificación. "Sistemas de información documental: concepto, modelo, estructura y organización”. En: López Yepes, José. Manual de ciencias de la información. Madrid: Pirámide, 2002, pp. 519-536.

Moreiro González, José Antonio; Navarro Bonilla, Diego; Esteban Navarro, Miguel Ángel [et al.]. Gestión del conocimiento y servicios de inteligencia. Madrid: Universidad Carlos III, 2004.

Ramos Simón, Luis Fernando. Dirección, administración y marketing de empresas e instituciones documentales. Madrid: Síntesis, 1995.

Lourdes Castelló Cogollos, Universitat de Valencia.

casco@alumni.uv.es 